Please do not remove this page

RMIT

UNIVERSITY

\title{
Factors influencing mortality among alcohol and drug treatment clients in Victoria, Australia: The role of demographic and substance use characteristics
}

Lloyd, Belinda; Barratt, Monica; Ferris, Jason; Best, David; Lubman, Dan

https://researchrepository.rmit.edu.au/esploro/outputs/9921861884901341/filesAndLinks?institution=61RMIT_INST\&index=null

Lloyd, B., Barratt, M., Ferris, J., Best, D., \& Lubman, D. (2013). Factors influencing mortality among alcohol and drug treatment clients in Victoria, Australia: The role of demographic and substance use characteristics. Australian and New Zealand Journal of Psychiatry, 47(9), 859-867.

https://doi.org/10.1177/0004867413491155

Document Version: Accepted Manuscript

Published Version: https://doi.org/10.1177/0004867413491155

Repository homepage: https://researchrepository.rmit.edu.au

(c) 2013 The Royal Australian and New Zealand College of Psychiatrists.

Downloaded On 2023/04/26 21:23:00 +1000 
Factors influencing mortality among alcohol and drug treatment clients in Victoria, Australia:

the role of demographic and substance use characteristics

Belinda Lloyd ${ }^{1,2}$, Monica J. Barratt ${ }^{1,3}$, Jason Ferris ${ }^{1,4}$, David Best ${ }^{1,2}$, Dan I. Lubman ${ }^{1,2}$

${ }^{1}$ Turning Point Alcohol \& Drug Centre, Eastern Health, Melbourne, Victoria, Australia

${ }^{2}$ Eastern Health Clinical School, Monash University, Melbourne, Victoria, Australia

${ }^{3}$ National Drug Research Institute, Curtin University, Melbourne, Victoria, Australia

${ }^{4}$ Institute for Social Science Research, University of Queensland, St Lucia, Queensland, Australia

Running head: Mortality predictors following drug treatment

\section{Declaration of interest}

Prof. Dan Lubman has received speaking honorarium for Astra Zeneca and Janssen, as well as travel support from Lundbeck.

\section{Corresponding Author:}

Dr Belinda Lloyd

Senior Research Fellow

Turning Point Alcohol and Drug Centre

54-62 Gertrude Street

Fitzroy Victoria 3065, Australia

belindal@turningpoint.org.au

(Ph) 0394129958

(Fax) 0394163420

Word count: 3495

Key words: Mortality, alcohol, drug, treatment, survival analysis, cohort, population 


\begin{abstract}
:
Aims: Clinical studies consistently identify alcohol- and drug-related treatment populations as more likely to die prematurely compared with age-matched general population. However, demographic characteristics and primary drug of concern as predictors of mortality risk following treatment have not been adequately explored. This paper examines relationships between substance use, demographic factors and mortality among alcohol and drug treatment clients.

Design, Setting and Participants: A retrospective cohort incorporating seven years of data was utilised to examine mortality outcomes in the two years following treatment among Victorian clients recorded on the Alcohol and Drug Information Service (ADIS) database by linking partial client identifiers with the National Death Index (NDI). A cohort of 18,686 clients engaged in at least one course of treatment over a 12-month period were included.
\end{abstract}

Measurement: Analysis of crude and standardised mortality rates across client groups in terms of presenting drug of concern for treatment and demographic characteristics.

Findings: Higher risk of premature death was associated with older age, being male, not being employed, living alone, medical and psychiatric comorbidity, recent injecting, and history of intensive drug treatment access. Alcohol treatment clients had the worst prognosis. After adjustment for client characteristics, alcohol treatment clients experienced a significantly higher rate of death compared with other clients.

Conclusions: Findings from these previously unexplored data highlight the need to increase awareness of the range and magnitude of risks associated with harmful alcohol use, and identify approaches to enhance treatment effectiveness to reduce negative outcomes following treatment for populations at elevated risk of harm. 


\section{Introduction}

People with alcohol- and drug-related problems in treatment are more likely to die prematurely when compared with age-matched peers in the general population [1-3]. Demographic factors that elevate the risk of death among alcohol and drug users identified previously include physical characteristics such as male gender [4-9] and older age [4,5,7,8,10,11], physical co-morbidities such as chronic medical conditions [11-13], psychiatric comorbidity $[1,12,13]$, homelessness $[1,13,14]$ and low social support $[10,11]$. Drug use characteristics have also been identified as risk factors for elevated risk of death, and include injecting drug use [8,14-16], polydrug use [1], and alcohol or heroin as substance of choice compared with cocaine [13]. Thus, both direct risks associated with the physiological effects of specific drugs (such as central nervous system (CNS) depressants like alcohol and heroin), and cumulative impacts of alcohol and drug use on health and related social factors contribute significantly to the risk of death among drug users.

Recent research examining mortality outcomes for people with a drug use history indicates that treatment engagement is protective against premature mortality [7,17]. Mortality rates among opioid pharmacotherapy clients were typically lower in treatment than after leaving treatment. For instance, Degenhardt et al. [7] found that opioid pharmacotherapy clients had an in-treatment crude mortality rate of 6.0 (95\% confidence interval (CI): 5.7-6.4) per 1000 person-years compared with an out-oftreatment rate of 11.5 (95\% CI: 11.1-12.0) per 1000 person-years. The first four weeks after commencing treatment and the first four week post treatment witness clients at an increased risk of drug related mortality, with elevated mortality risk among opioid pharmacotherapy clients in the first two to four weeks after ceasing treatment $[6,7]$. The first one to four weeks of induction into treatment is also a time of elevated risk [7,18]. Less is known about longer-term mortality outcomes following treatment that does not involve opioid pharmacotherapy or for clients who have sought treatment for alcohol or other drugs. Given the high proportions of clients seeking treatment for alcohol use or other non-opioid substances from specialist alcohol and drug agencies [19], there is a need to examine 
longer term outcomes following alcohol and drug treatment across different presenting drugs of concern.

Despite numerous studies demonstrating lower mortality outcomes for those engaged in drug treatment, the role of demographic characteristics and primary drug of concern as predictors of longerterm mortality risk in the two years following treatment has not been adequately explored. This paper helps addresses this gap by examining the relationship between substance use characteristics and demographic factors and mortality outcomes using a population level cohort of drug treatment clients across a range of treatment modalities outside of general practice-based pharmacotherapy in Victoria, Australia.

\section{Method:}

Retrospective cohort data were utilised to examine mortality outcomes among Victorian clients. Client data was drawn from the Alcohol and Drug Information Service (ADIS) database (including detailed information regarding all specialist treatment occurring in Victoria) and this was linked to the National Death Index (NDI; which includes all detailed information regarding cause of death for all deaths occurring in Australia). Linkage between the two databases was established by linking partial client identifiers between the two databases.

\section{$\underline{\text { Cohort }}$}

The ADIS database is a register of government-funded, specialist Alcohol and Other Drug (AOD) treatment services. Members of the cohort for analysis had to have completed at least one course of AOD treatment (for example, counselling, residential withdrawal) in the 12-month period between 1 July 2000 and 30 June 2001, with their first course of treatment (COT) starting on or after 1 January 2000. Records were only included in the cohort if they had a valid date of birth and start date of their 
first COT. Based on these criteria our cohort consisted of 18,686 clients. To construct a unique identifier we used a unique combination of partial name identifiers (second 2 letters of first name and first 2 letters and last letter of surname), date of birth and gender (for example John Doe, 17/01/1969, male would be ohdoe $170169 \mathrm{~m}$ ). These codes were used to identify cohort individuals and link their information across ADIS and NDI.

\section{$\underline{\text { Data sources }}$}

$A D I S$

Cohort codes were matched across 8 years of data from ADIS, including COTs that terminated between 1/7/2000 and 30/6/2008. Multiple COTs were common among the cohort with the median of 2 (IQR 1-5) COT. COTs could be continuous, indicating a change of treatment type, agency or drug of concern. Multiple COTs could be sequential, overlapping or embedded within other COTs, e.g. a short episode of outreach could occur within a 6-month long course of counselling.

COTs were excluded if they started before 1 January 2000 or after 1 January 2007 . The total number of COTs provided to the cohort was 89,764. A series of data extraction algorithms were applied to clean and prepare the data, including amendment of overlapping COTs and recoding multiple consecutive COTs. After cleaning, approximately $15 \%$ of records were removed; the total number of COTs provided was 76,342 .

\section{National Death Index (NDI)}

The cohort codes extracted from ADIS were submitted to the Australian Institute of Health and Welfare (AIHW) for data matching with NDI. AIHW ran three linkage passes. The first involved using an exact match unique identifier. The second pass was the same as the first except matched on only month and year of birth. The third involved cases where the client had been recorded validly as deceased within ADIS. Where a match occurred death had to have occurred after the date of last contact in ADIS. 
Almost all $(532 ; 94 \%)$ deaths were linked using the exact method with NDI. A further 10 clients (2\%) were linked to NDI records using the second pass method and the remaining 23 (4\%) deaths were linked using the third pass method.

Once NDI and ADIS data were combined, causes of death were examined. In NDI data, one underlying cause of death and up to 11 secondary causes were recorded using ICD-10 coding conventions. Generally, only underlying cause of death was used to categorise deaths, apart from opioid deaths that were cross-classified with particular secondary death codes. A standard mortality toolkit was used to classify deaths into categories [20]. Another category of overdose-related deaths was also constructed based on existing protocols [21,22].

\section{$\underline{\text { Analysis }}$}

Data were examined using survival analysis, and conducted using Stata 11.

\section{Predictor variables}

Demographic, drug and treatment variables available in ADIS were included as predictors in survival time analysis. Static demographic variables included sex, country of birth (born in Australia or not) and indigenous status. All other demographic variables were time-varying covariates and included age, employment status (employed or not employed), living status (alone or with family/others), temporary or homeless accommodation status, and current involvement in the justice system (through community based orders, parole, bail, custody, etc.). Primary drug of concern and injecting drug use were measured at the start of each COT. Medical and psychiatric comorbidities were also included as covariates. Treatment-related variables included type of treatment received, number of COTs per client and reason for treatment termination. 
As a proxy measure of polydrug use, individuals who had received multiple COTs with different primary drugs of concern were classified as polydrug users. While this provides a conservative measure of polydrug use, it has utility in identifying clients with multiple drugs of concern requiring treatment.

\section{Crude mortality rates and standardised mortality ratios}

To calculate crude mortality rates (CMRs) and standardised mortality ratios (SMRs), time at risk (in person-years) was calculated from date of first contact with ADIS (between 1 January 2000 and 30 June 2001) to either date of death or for right-censored cases, the earliest of 2 years after the last COT ended or 31 December 2006. All-cause CMRs were calculated by dividing total number of deaths by the equivalent sum of person-years of observation. CMR are presented per 1000 person-years (PY). Indirect all-cause SMRs were calculated by 10 -year age groups based on death rates of the Victorian population in the year 2000. Two-sided $95 \%$ confidence intervals (95\% CI) were based on Poisson distribution.

\section{Factors predicting mortality after treatment discharge}

As some cohort members either died during treatment or were in treatment at the end of the observation period, the number in the cohort used to predict mortality after treatment discharge was reduced from 18,686 to 17,861 ( 825 observations deleted) which reduced the number of deaths from 570 to 423 - the decrease reflecting high risk of death during early stages of treatment. Time at risk was calculated from the date of termination of last COT to death or censorship. Censorship occurred at the earliest of 2 years after last COT ended or 31 December 2006. The median survival time was 2 years.

Cox proportional hazards models were run to assess bivariate relationships between indicator variables and time to death. Variables that did not meet the proportional hazards assumption [23] in bivariate models were interacted with time (defined as the first and second years of follow-up) by entering two 
'heaviside' functions into models. When heaviside functions are used, fixed hazard ratios for specified time intervals are generated [24]. Proportional hazards assumptions were tested again with these timeinteracted variables and all models were well-specified.

Only covariates with p-values $<0.05$ in univariate models were included as controls in the multivariate Cox proportional hazards models. These models controlled for age, sex, not being employed, living alone, medical comorbidity, psychiatric comorbidity, recent injecting and total number of COTs received. Unadjusted and adjusted models were run separately for each drug of concern, with the reference group defined as all other cases.

\section{Results}

\section{Table 1}

Treatment data of 18,686 individuals was available for analysis. This represents 69,270 PY over 89,764 COTs. Two thirds (65\%) of the cohort were male and the median age on commencement of treatment was 28 years (IQR 21-36). Heroin and other opioids and alcohol were the most commonly reported primary drugs of concern on entry into a COT within the cohort. Heroin and other opioids accounted for $42 \%$ of COTs with a known drug of concern, and alcohol accounted for a further third (Table 1).

\section{Table 2}

Overall, the cohort was followed up for a median of 2.8 years (IQR 2.1-5.7) or a total of 69,270 PY. In that period there were 570 deaths, giving an overall CMR of 8.2 deaths per 1000 PY (95\% CI: 7.68.9; Table 2). The mortality rate among this cohort was 6.8 times the SMR of the age-matched Victorian population (95\% CI: 6.3-7.4). CMRs were significantly higher among males, although SMRs did not significantly differ (Table 2). CMRs increased with age, in contrast to SMRs which 
decreased with age. For all ages, higher mortality rates were noted for treatment clients compared with the age-matched Victorian population.

Mortality associated with specific drug types were calculated by summing the person-years and number of deaths related to any client coded with that primary drug of concern (Table 2). Among clients with cannabis as a primary drug of concern the CMR was 4.4 (95\% CI: 3.6-5.5) times higher than the age-matched Victorian population. The CMR for alcohol (11.1; 95\% CI: 9.9-12.4) was significantly higher than heroin and other opioids (7.1; 95\% CI: 6.3-8.0), however, SMRs were not significantly different for alcohol and heroin and other opioids (7.0 vs 7.7). The highest SMR was associated with volatile substances - among clients with volatile substances as a primary drug of concern the SMR was 11.3 (95\% CI: 6.1-21.1) times higher than the age-matched Victorian population.

\section{Client characteristics and risk of death}

\section{Table 3}

Of the 18,686 unique clients available for analysis, 678 clients were in treatment at the end of the study period (December 2006) and 147 clients died whilst in treatment. Data from these clients are not included in further analysis. Table 3 explores the characteristics of 17,861 clients representing 423 deaths. Characteristics associated with a significantly increased risk of death after adjustment for the presence of other factors included older age, being male, not being employed, living alone, medical comorbidity, psychiatric comorbidity, recent injecting drug use and a history of intensive drug treatment service access (multiple COTs). Recent injecting drug use on entry into treatment significantly increased the hazard of death by 55 per cent (95\% CI: 13-111\%) in the first year of follow-up, but not in the second year. 


\section{Drug of concern and risk of death in the first and second year following treatment}

\section{Table 4 and Figure 1}

After adjustment for covariates, cohort members whose last COT was for alcohol experienced a significantly higher rate of death compared with all others in the cohort: the hazard of death increased by 62 per cent (95\% CI: $20-118 \%$ ) in the first year of follow-up and 123 per cent (95\% CI: $41-251 \%$ ) in the second year of follow-up (Table 4 and Figure 1). Cohort members whose last COT was for cannabis experienced a significantly lower rate of death: the hazard decreased by 57 per cent $(95 \% \mathrm{CI}$ : 34-72\%) across the follow-up period. The risk of death for clients whose last COT was for benzodiazepines, sedatives or hypnotics increased by 69 per cent in the first year of follow-up compared with the rest of the sample, however this was not statistically significant. No deaths attributable to benzodiazepines, sedatives or hypnotics during the second year of follow-up were indicated.

\section{Discussion}

This paper reports CMRs for a cohort of Victorian clients who sought and engaged in specialist treatment for a wide range of drug types. Our all-cause CMR for opioid users of 7.1 (6.3-8.0) per 1000 PY was lower than the rate measured in the largest study of Australian opioid treatment clients in pharmacotherapy, who died at a rate of 8.9 (8.6-9.2) per 1000 PY [7]. In Victoria, most opioid pharmacotherapy treatment is provided by general practitioners, and is not captured in the data presented here. The lower mortality rate among the opioid users in the present study may be due to the cohort being drawn from treatment populations outside of the primary pharmacotherapy system and engaged in other forms of treatment, or a combination of pharmacotherapy and other treatment modalities. This may in turn mean that this study findings reflects a cohort experiencing less negative health consequences impacting upon mortality compared with other studies that have cohorts of 
pharmacotherapy clients, and also differences in different treatment system designs $[1,6,7,25]$. This distinct population of clients represents a cohort likely to be fundamentally different from opioid pharmacotherapy clients in terms of drug use, consistent treatment engagement, broader service engagement and other characteristics.

The mortality rate from the largest study of drug users accessing treatment in Europe [15] found 8.2 drug-related deaths per 1000 PY of follow-up. While this rate was the same as the overall rate found in the current study, our overall rate included all causes of mortality, not just drug-related. On this evidence, our estimates are likely to indicate a lower rate of premature death in the Victorian cohort compared with the European cohort, and with all other international cohorts reviewed in the introduction. These rates do largely accord with comparable Australian rates where available. One of the contributing factors for lower mortality rates in this cohort than those reported in a number of international studies is likely to be the low rates of HIV infection among illicit drug users in Australia, while the selection of a sample of clients not primarily engaged in pharmacotherapy treatment is another factor that will have influenced mortality rates. Exploration in differences in cause of death may also yield findings that could reflect population differences in risk and harm.

As predicted by previous research, higher risk of premature death among this cohort was associated with older age, being male, not being employed, living alone, medical and psychiatric comorbidity and recent injecting. Having a history of intensive drug treatment service access (multiple COTs) - which is likely to reflect greater severity of complexity of substance use and related issues - also significantly increased the risk of premature death. In contrast to previous findings, being homeless (defined in these data as not having access to safe, secure and adequate housing) was not associated with significantly increased mortality risk.

Most significantly, clients discharged from alcohol treatment had the worst prognosis over the 2-year follow-up period. After adjustment for client characteristics, cohort members whose last COT was for 
alcohol continued to experience a significantly higher rate of death compared with all others in the cohort. These findings add to those of a US cohort of treatment clients followed after discharge, for whom alcohol and heroin clients were at greater risk than cocaine clients [13].

Clients discharged from cannabis treatment experienced a significantly lower rate of death. It is important to note, however, that CMRs for clients following treatment for cannabis use were significantly elevated when compared with age-matched general population peers - highlighting a population under-represented in the literature in terms of evidence based intervention developed and evaluated with robust outcome measures that meaningfully assess effectiveness of treatment, but potentially at risk of elevated harms.

Clients who had engaged in treatment in relation to volatile substance use were found to have the highest rate of death. This may reflect the younger age of many volatile substance users, and also a combination of factors that increase complexity of case presentation, and therefore increased risk of premature death. Further exploration of factors that influence outcomes of this high risk population are needed to disentangle to relationships between substance use and other factors that contribute to harm for this population.

\section{$\underline{\text { Limitations }}$}

This study has three main limitations: the accuracy and completeness of linkage methodology, the absence of key variables in ADIS, and the size of the extracted cohort group.

Firstly, the completeness and accuracy of the data linkage between ADIS and NDI was limited by the use of deterministic methods with partial client identifiers. While it is likely that deterministic linkage methods underestimate mortality by missing clients who use nicknames or who have incomplete data, more evidence is needed regarding the extent to which linkage using partial identifiers underestimates rates. To improve quality, other information from ADIS could potentially be added into the linkage 
probability mix, including postcode of client residence. Despite this, the linkage process yielded robust results, and a sufficiently large cohort to enable analysis of factors influencing mortality among this population.

Secondly, key variables were not recorded in ADIS which are likely to influence the survival of drug treatment clients. Some variables in ADIS, like polydrug use, were available but were not used due to incompleteness. Other potentially useful variables in ADIS, such as significant treatment goal achievements, are not consistently collected. Other variables that have been shown as important covariates for mortality among drug users that were not available include age of initiation into drug use and age of first injection [15]. Furthermore, coding practices for predictor variables may have changed systematically over the time period examined as a result of policy changes. For example, additional drugs of concern, and different categories of existing drugs of concern, have been adopted during this time. To minimise the impact on results, only complete and consistently accurate variables were included in the analyses.

Another important issue is that most pharmacotherapy treatment provided in Victoria is not recorded in the ADIS dataset, as the pharmacotherapy treatment system in Victoria primarily involves treatment provided by general practitioners rather than specialist AOD treatment services. Accordingly, this study differs from most treatment mortality research in the addiction field, where the primary client population examined tends to consist of clients seeking treatment for opioid dependence in the form of opioid substitution pharmacotherapy. Whilst this may be seen as a limitation in terms of comparability of findings, this research provides an insight into diverse AOD treatment populations.

\section{Conclusion}

There is little international evidence examining mortality among populations of drug users following treatment engagement and cessation across multiple drugs of concern, and less is known in an Australian context. Whilst previous research has focused primarily on heroin and other opioid users 
engaging in pharmacotherapy treatment as a population perceived to be at greatest risk of premature death, this study has found that, after controlling for a range of demographic, health and drug use characteristics, risk of premature death is greatest for patients whose primary drug of concern is alcohol - with risk of death increasing with time since cessation of treatment. These findings highlight the need to increase awareness in the community as well as among clinicians of the range and magnitude of risks associated with harmful alcohol use patterns. Furthermore, the elevated risk of harm for clients following treatment for cannabis use compared with the general population reflects a group not well understood in terms of factors that influence risk of harm, or health and social benefits following treatment. There is an urgent need to identify risk and protective factors relevant to treatment engagement, treatment delivery and reduction of negative outcomes following cessation of treatment that support recovery and global improvements in health and social functioning for patients beyond the treatment episode.

\section{Acknowledgements}

The authors would like to acknowledge and kindly thank the Australian Institute of Health and Welfare for access to NDI data, and the Victorian Department of Health for access to ADIS data. This project was funded by the Victorian Department of Health. 


\section{References}

1. Gossop, M, Stewart, D, Treacy, S, Marsden, J. A prospective study of mortality among drug misusers during a 4-year period after seeking treatment. Addiction 2002, 97(1), 39-47.

2. Oppenheimer, E, Tobutt, C, Taylor, C, Andrew, T. Death and survival in a cohort of heroin addicts from London clinics: A 22-year follow-up study. Addiction 1994, 89(10), 1299-1308.

3. Warner-Smith, M, Lynskey, M, Hall, W, Monteiro, M. Challenges and approaches to estimating mortality attributable to the use of selected illicit drugs. European Addiction Research 2001, 7(3), 104-116.

4. Bell, J, Trinh, L, Butler, B, Randall, D, Rubin, G. Comparing retention in treatment and mortality in people after initial entry to methadone and buprenorphine treatment. Addiction 2009, 104(7), 1193 1200.

5. Bird, SM, Hutchinson, SJ, Goldberg, DJ. Drug-related deaths by region, sex, and age group per 100 injecting drug users in Scotland, 2000-01. Lancet 2003, 362(9388), 941-944.

6. Clausen, T, Anchersen, K, Waal, H. Mortality prior to, during and after opioid maintenance treatment (OMT): A national prospective cross-registry study. Drug and Alcohol Dependence 2008, 94(1), 151-157.

7. Degenhardt, L, Randall, D, Hall, W, Law, M, Butler, T, Burns, L. Mortality among clients of a state-wide opioid pharmacotherapy program over 20 years: Risk factors and lives saved. Drug and Alcohol Dependence 2009, 105(1-2), 9-15.

8. Hickman, M, Carnwath, Z, Madden, P, Farrell, M, Rooney, C, Ashcroft, R, et al. Drug-related mortality and fatal overdose risk: Pilot cohort study of heroin users recruited from specialist drug treatment sites in London. Journal of Urban Health 2003, 80(2), 274-287.

9. Ravndal, E, Amundsen, EJ. Mortality among drug users after discharge from inpatient treatment: An 8-year prospective study. Drug and Alcohol Dependence 2010, 108(1-2), 65-69.

10. Lewis, CE, Smith, E, Kercher, C, Spitznagel, E. Predictors of mortality in alcoholic men: A 20year follow-up study. Alcoholism: Clinical and Experimental Research 1995, 19(4), 984-991. 
11. Moos, RH, Brennan, PL, Mertens, JR. Mortality rates and predictors of mortality among latemiddle-aged and older substance abuse patients. Alcoholism: Clinical and Experimental Research 1994, 18(1), 187-195.

12. McCowan, C, Kidd, B, Fahey, T. Factors associated with mortality in Scottish patients receiving methadone in primary care: Retrospective cohort study. BMJ 2009, 338, b2225.

13. Saitz, R, Gaeta, J, Cheng, DM, Richardson, JM, Larson, MJ, Samet, JH. Risk of mortality during four years after substance detoxification in urban adults. Journal of Urban Health 2007, 84(2), $272-$ 282.

14. Langendam, MW, van Brussel, GH, Coutinho, RA, van Ameijden, EJ. The impact of harmreduction-based methadone treatment on mortality among heroin users. American Journal of Public Health 2001, 91(5), 774-780.

15. Bird, SM. Over 1200 drugs-related deaths and 190,000 opiate-user-years of follow-up: Relative risks by sex and age group. Addiction Research and Theory 2010, 18(2), 194-207.

16. Quan, VM, Vongchak, T, Jittiwutikarn, J, Kawichai, S, Srirak, N, Wiboonnatakul, K, et al. Predictors of mortality among injecting and non-injecting HIV-negative drug users in northern Thailand. Addiction 2007, 102(3), 441-446.

17. Darke, S, Mills, KL, Ross, JE Teesson, M, Rates and correlates of mortality amongst heroin users: Findings from the Australian Treatment Outcome Study (ATOS), 2001-2009. Drug and Alcohol Dependence 2011, 115(3), 190-195.

18. Buster, MC, van Brussel, GH, van den Brink, W. An increase in overdose mortality during the first 2 weeks after entering or re-entering methadone treatment in Amsterdam. Addiction 2002, 97(8), 9931001.

19. AIHW. Alcohol and other drug treatment services in Australia 2009-10: report on the National Minimum Data Set. 2011, Drug treatment series no. 14. Cat. no. HSE 114. Canberra: AIHW. 20. Randall, D, Roxburgh, A, Gibson, A, Degenhardt, L. Mortality among people who use illicit drugs: A toolkit for classifying major causes of death 2009, NDARC Technical Report No. 301. Sydney: National Drug and Alcohol Research Centre, University of New South Wales. 
21. Stoové, MA, Dietze, PM, Aitken, CK, Jolley, D. Mortality among injecting drug users in Melbourne: A 16-year follow-up of the Victorian Injecting Cohort Study (VICS). Drug and Alcohol Dependence 2008, 96(3), 281-285.

22. Stoové, MA, Dietze, PM, Jolley, D. Overdose deaths following previous non-fatal heroin overdose: Record linkage of ambulance attendance and death registry data. Drug and Alcohol Review $2009,28(4), 347-352$.

23. Hosmer, DW, Lemeshow, S. Applied survival analysis: Regression modelling of time to event data. 1999, John Wiley and Sons, New York.

24. Kleinbaum, DG, Klein, M. Survival analysis. A self-learning text (2nd ed.). 2005, Springer: New York.

25. Gibson, A, Degenhardt, L, Mattick, RP, Ali, R, White, J, O'Brien, S. Exposure to opioid maintenance treatment reduces long-term mortality. Addiction 2008, 103(3), 462-468. 
Table 1: Primary drug of concern across 89,764 COTs - 2000 to 2007

\begin{tabular}{lrrr}
\hline Drug type & Frequency & \% of total & \% of known \\
\hline Heroin and other opioids & 37,456 & 41.7 & 42.1 \\
Alcohol & 28,596 & 31.9 & 32.1 \\
Cannabis & 13,603 & 15.2 & 15.3 \\
Amphetamine & 4,541 & 5.1 & 5.1 \\
Benzodiazepines, sedative and hypnotics & 2,538 & 2.8 & 2.9 \\
Other psychostimulants and hallucinogens & 414 & 0.5 & 0.5 \\
Volatile substances & 741 & 0.8 & 0.8 \\
Nicotine & 355 & 0.4 & 0.4 \\
Other & 775 & 0.9 & 0.9 \\
Total known & 89,019 & 99.2 & 100.0 \\
Unknown & 745 & 0.8 & \\
Total & 89,764 & 100.0 & \\
\hline
\end{tabular}


Table 2: Crude mortality rates and standardised mortality ratios by sex, age group and primary drug of concern $(\mathrm{N}=18,686)$

\begin{tabular}{|c|c|c|c|c|c|c|c|}
\hline & Person-years & $\begin{array}{l}\text { Observed } \\
\text { deaths }\end{array}$ & CMR & $95 \% \mathrm{Cl}$ & $\begin{array}{c}\text { Expected } \\
\text { deaths }\end{array}$ & SMR & $95 \% \mathrm{Cl}$ \\
\hline \multicolumn{8}{|l|}{ Sex } \\
\hline Males & 45063 & 427 & 9.5 & $8.6-10.4$ & 70 & 6.1 & $5.6-6.7$ \\
\hline Females & 24207 & 143 & 5.9 & $5.0-7.0$ & 21 & 6.7 & $5.7-7.9$ \\
\hline \multicolumn{8}{|l|}{ Age group } \\
\hline Less than 20 years & 6196 & 17 & 2.7 & $1.7-4.4$ & 2 & 8.7 & $5.4-14.1$ \\
\hline $20-29$ years & 28014 & 161 & 5.7 & $4.9-6.7$ & 21 & 7.8 & $6.7-9.2$ \\
\hline $30-39$ years & 20706 & 145 & 7.0 & $6.0-8.2$ & 20 & 7.1 & $6.1-8.4$ \\
\hline $40-49$ years & 10287 & 140 & 13.6 & $11.5-16.1$ & 16 & 8.6 & $7.3-10.2$ \\
\hline $50-59$ years & 3233 & 72 & 22.3 & $17.7-28.1$ & 12 & 5.9 & $4.7-7.4$ \\
\hline $60-69$ years & 671 & 23 & 34.3 & $22.8-51.6$ & 7 & 3.3 & $2.2-5.0$ \\
\hline $70-79$ years & 157 & 10 & 63.8 & $34.3-118.5$ & 5 & 2.2 & $1.2-4.0$ \\
\hline $80+$ years & 6 & 2 & 310.2 & $77.6-1,240.3$ & 1 & 3.0 & $0.8-12.2$ \\
\hline \multicolumn{8}{|l|}{ Primary drug of concern (any COT) } \\
\hline Alcohol & 28970 & 321 & 11.1 & $9.9-12.4$ & 46 & 7.0 & $6.3-7.9$ \\
\hline Cannabis & 21199 & 79 & 3.7 & $3.0-4.6$ & 18 & 4.4 & $3.6-5.5$ \\
\hline Amphetamine & 9267 & 43 & 4.6 & $3.4-6.3$ & 8 & 5.3 & $3.9-7.1$ \\
\hline Other psychostimulants and hallucinogens & 1223 & 7 & 5.7 & $2.7-12.0$ & 1 & 6.6 & $3.2-13.9$ \\
\hline Benzodiazepines, sedatives and hypnotics & 5058 & 40 & 7.9 & $5.8-10.8$ & 6 & 6.2 & $4.5-8.4$ \\
\hline Nicotine & 809 & 3 & 3.7 & $1.2-11.5$ & 1 & 2.8 & $0.9-8.7$ \\
\hline Volatile substances & 1281 & 10 & 7.8 & $4.2-14.5$ & 1 & 11.3 & $6.1-21.1$ \\
\hline Other drugs & 2103 & 11 & 5.2 & $2.9-9.4$ & 3 & 3.5 & $1.9-6.4$ \\
\hline Overall cohort & 69270 & 570 & 8.2 & $7.6-8.9$ & 83 & 6.8 & $6.3-7.4$ \\
\hline
\end{tabular}

Note. All rates per 1,000 person-years. Person-years can be assigned to multiple drugs of concern. 
Table 3: Client characteristics and associated risk of death $(\mathrm{N}=17,861)$

\begin{tabular}{|c|c|c|c|c|c|}
\hline Characteristic & Percentage & $\begin{array}{l}\text { Unadjusted } \\
\text { hazard ratio }\end{array}$ & $95 \% \mathrm{Cl}$ & $\begin{array}{c}\text { Adjusted } \\
\text { hazard ratio a }\end{array}$ & $95 \% \mathrm{Cl}$ \\
\hline Median age at start (IQR) & $28(21-36)$ & $1.05^{* * *}$ & $1.04-1.06$ & $1.05^{* * *}$ & $1.03-1.06$ \\
\hline Male & 65 & $1.60^{* * *}$ & $1.28-1.99$ & $1.57^{* * *}$ & $1.22-2.03$ \\
\hline Not born in Australia & 13 & 1.15 & $0.87-1.52$ & 1.00 & $0.73-1.36$ \\
\hline Indigenous & 6 & 0.97 & $0.63-1.50$ & 1.38 & $0.88-2.16$ \\
\hline Not employed & 77 & $1.98^{* * *}$ & $1.50-2.62$ & 1.68 ** & $1.21-2.33$ \\
\hline Lives alone & 21 & $2.13^{* * *}$ & $1.73-2.61$ & $1.39 * *$ & $1.09-1.77$ \\
\hline Temporary or no accommodation & 15 & 1.11 & $0.86-1.45$ & 1.05 & $0.76-1.45$ \\
\hline Justice system involvement & 42 & 0.89 & $0.73-1.08$ & 1.02 & $0.80-1.31$ \\
\hline Medical comorbidity & 22 & $2.25^{* * *}$ & $1.84-2.76$ & $1.54^{* * *}$ & $1.22-1.96$ \\
\hline Psychiatric comorbidity & 23 & $1.58^{* * *}$ & $1.28-1.96$ & 1.30 * & $1.02-1.67$ \\
\hline Injected in last 3 months ${ }^{b}$ & 24 & & & & \\
\hline In Year 1 & & 1.17 & $0.90-1.54$ & $1.55^{* *}$ & $1.13-2.11$ \\
\hline In Year 2 & & 1.12 & $0.75-1.67$ & 1.33 & $0.83-2.15$ \\
\hline $\begin{array}{l}\text { Multiple primary drugs of concern } \\
\text { Number of COTs }\end{array}$ & 31 & 1.04 & $0.84-1.28$ & 0.90 & $0.68-1.18$ \\
\hline 1 & 34 & 1.00 & & & \\
\hline $2-4$ & 36 & $1.44^{* *}$ & $1.13-1.83$ & 1.52 * & $1.10-2.09$ \\
\hline $5+$ & 30 & $2.14^{* * *}$ & $1.67-2.73$ & $2.32^{* * *}$ & $1.65-3.27$ \\
\hline
\end{tabular}

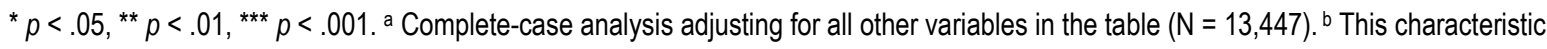
did not meet the proportional hazards assumption and therefore was stratified by follow-up year. 
Table 4: Most recent drug of concern and associated risk of death $(\mathrm{N}=17,682)$

\begin{tabular}{|c|c|c|c|c|c|}
\hline Characteristic & $\begin{array}{l}\text { Per } \\
\text { cent }\end{array}$ & $\begin{array}{l}\text { Unadjusted } \\
\text { hazard ratio }\end{array}$ & $95 \% \mathrm{Cl}$ & $\begin{array}{c}\text { Adjusted } \\
\text { hazard ratio a }\end{array}$ & $95 \% \mathrm{Cl}$ \\
\hline Heroin and other opioids & 35 & 0.85 & $0.69-1.04$ & 0.99 & $0.76-1.29$ \\
\hline Alcohol b & 34 & & & & \\
\hline In Year 1 & & $2.18^{* * *}$ & $1.72-2.75$ & 1.62 ** & $1.20-2.18$ \\
\hline In Year 2 & & $2.75^{\star * *}$ & $1.96-3.86$ & $2.23^{* * *}$ & $1.41-3.51$ \\
\hline Cannabis & 20 & $0.29 * * *$ & $0.20-0.42$ & $0.43^{* * *}$ & $0.28-0.66$ \\
\hline Amphetamine ${ }^{b}$ & 5 & & & & \\
\hline In Year 1 & & 0.62 & $0.32-1.21$ & 0.75 & $0.38-1.47$ \\
\hline In Year 2 & & 0.15 & $0.02-1.06$ & 0.20 & $0.03-1.41$ \\
\hline $\begin{array}{l}\text { Other psychostimulants \& } \\
\text { hallucinogens }\end{array}$ & 1 & 0.29 & $0.04-2.09$ & $\mathrm{~N} / \mathrm{A}$ & \\
\hline 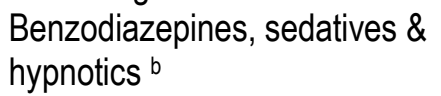 & 3 & & & & \\
\hline In Year 1 & & 1.96 * & $1.15-3.36$ & 1.69 & $0.96-2.98$ \\
\hline In Year 2 & & $N / A$ & & & \\
\hline Nicotine & 1 & 0.33 & $0.05-2.36$ & 0.57 & $0.08-4.10$ \\
\hline Volatile substances & 1 & 0.38 & $0.05-2.73$ & 0.67 & $0.09-4.76$ \\
\hline Other & 1 & 0.48 & $0.15-1.49$ & 0.61 & $0.19-1.90$ \\
\hline
\end{tabular}

Note. ${ }^{*} p<.05,{ }^{* \star} p<.01,{ }^{* * *} p<.001$. a Complete-case analysis adjusted for age, gender, not employed, lives alone, psychiatric comorbidity, medical comorbidity, recent drug injection and total COTs. ${ }^{b}$ Variables that did not meet the proportional hazards assumption were stratified by follow-up year. N/A Analysis not available as 0 deaths were attributable to the particular drug (in the particular time period). 
Figure 1: Kaplan-Meier estimates by selected drugs of concern: Heroin and other opiates, alcohol, cannabis

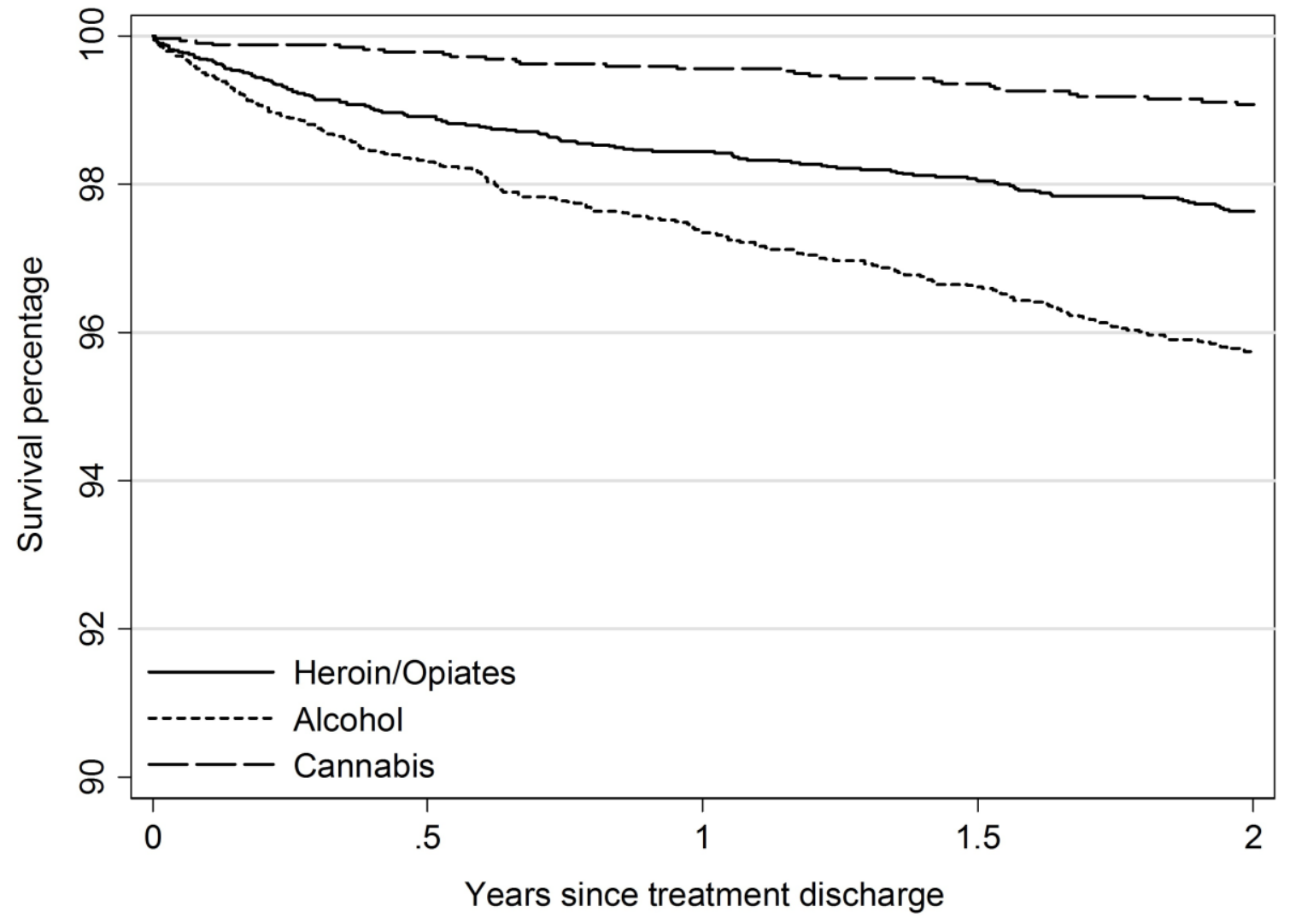

